\title{
Prescription and dosing of urate-lowering therapy, rather than patient behaviours, are the key modifiable factors associated with targeting serum urate in gout
}

Nicola Dalbeth ${ }^{1 *}$, Meaghan E House ${ }^{1}$, Anne Horne ${ }^{1}$, Keith J Petrie², Fiona M McQueen ${ }^{3}$ and William J Taylor ${ }^{4}$

\begin{abstract}
Background: Long term serum urate (SU) lowering to a target of $<0.36 \mathrm{mmol} / \mathrm{l}(6 \mathrm{mg} / \mathrm{dl})$ is recommended for effective gout management. However, many studies have reported low achievement of SU targets. The aim of this cross-sectional study was to examine the clinical and psychological factors associated with SU targets in patients with gout.

Methods: Patients with gout for $<10$ years were recruited from primary and secondary care settings. SU target was defined as SU concentration $<0.36 \mathrm{mmol} / \mathrm{L}$ at the time of the study visit. Both clinical and psychological factors associated with SU target were analysed. The relationship between SU target and measures of gout activity such as flare frequency, tophi, work absences, and Health Assessment Questionnaire-II was also analysed.

Results: Of the 273 patients enrolled into the study, 89 (32.6\%) had SU concentration $<0.36 \mathrm{mmol} / \mathrm{L}$. Uratelowering therapy (ULT) use was strongly associated with SU target $(p<0.001)$. In those patients prescribed ULT $(n=181)$, allopurinol dose, patient confidence to keep SU under control, female sex, and ethnicity were independently associated with SU target. Other patient psychological measures and health-related behaviours, including adherence scores, were not independently associated with SU target in those taking ULT. Creatinine clearance, diuretic use, age, and body mass index were not associated with SU target. Patients at SU target reported lower gout flare frequency, compared with those not at target $(p=0.03)$.
\end{abstract}

Conclusions: ULT prescription and dosing are key modifiable factors associated with achieving SU target. These data support interventions focusing on improved use of ULT to optimise outcomes in patients with gout.

Keywords: Gout, Urate, Target, Allopurinol

\section{Background}

Gout is characterised by deposition of monosodium urate (MSU) crystals in the joints and other tissues. The central strategy for management of gout is long term serum urate (SU) lowering, which leads to dissolution of MSU crystals, prevention of acute gout flares, and regression of tophi [1]. A number of studies have demonstrated that long term SU lowering below a target of $0.36 \mathrm{mmol} / \mathrm{l}$ $(6 \mathrm{mg} / \mathrm{dl})$ is required to achieve these outcomes [2-5].

\footnotetext{
* Correspondence: n.dalbeth@auckland.ac.nz

${ }^{1}$ Department of Medicine, Faculty of Medical and Health Sciences, University of Auckland, 85 Park RdGrafton, Auckland 1023, New Zealand

Full list of author information is available at the end of the article
}

Based on these studies, a 'treat to serum urate target' approach has been recommended in a number of gout treatment guidelines [6,7]. However, studies from many countries have shown that gout management is frequently poor, with low achievement of treatment targets [8-11]. A variety of factors may contribute to undertreatment of gout [12]. Potential patient factors include medical co-morbidities, concomitant medications, attitudes to disease and therapy, and poor adherence to urate-lowering therapy (ULT) $[10,13,14]$. Medical practitioner factors may include prescription and adequate dosing of ULT [8]. To date, these factors have not been systematically explored in relation to achieving optimal

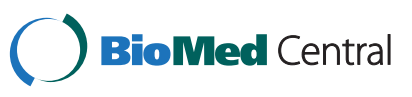


SU targets. Understanding these factors is important to guide treatment strategies to improve management of gout. The aim of this study was to examine the clinical and psychological factors associated with SU target $<0.36 \mathrm{mmol} / \mathrm{L}$ in patients with gout.

\section{Methods}

Patients with gout for less than 10 years were recruited by community advertising and through primary and secondary care clinics in Auckland and Wellington, New Zealand. As in other regions of the world, gout management is frequently suboptimal in New Zealand $[8,15]$. The inclusion criteria for this study were: a previous physician diagnosis of acute gout as defined by the Wallace classification for acute gout [16]; first attack of gout within the last 10 years; and ability to complete forms in English and provide written informed consent. Patients did not have an acute flare at the time of their study visit. The New Zealand Multi Regional Ethics Committee approved this study.

Participants attended a study visit which included a comprehensive clinical assessment. The following data were recorded: demographic data (age, gender, ethnicity), gout history (confirmation of diagnosis, disease duration, frequency of gout flares, gout treatments), medical history, examination, questionnaires (including Health Assessment Questionnaire (HAQ)-II, Brief Illness Perception Questionnaire (B-IPQ) [17], confidence in gout treatment questionnaire and a medication adherence score related to ULT (completed only by those patients taking ULT) [17]), and laboratory tests (including SU and creatinine).

Patient self-reported confidence in gout treatment was assessed using 0 (not at all confident)-10 (very confident) Likert scales, which covered aspects of gout management including confidence to follow recommended diet, take ULT regularly, have blood tests and keep blood urate level under control. These items were adapted from a diabetes-specific Multidimensional Diabetes Questionnaire [18].

Data were analysed using SPSS (v19, SPSS Inc., Chicago, IL). Means with standard deviations (SD) and percentages were used to describe the clinical characteristics of participants. SU target was defined as SU concentration $<0.36 \mathrm{mmol} / \mathrm{L}$ at the time of the study visit. T-tests and Chi square analysis was used to compare the group of patients at SU target with those who were not. Forward stepwise logistic regression analysis was used to determine the independent variables associated with SU target in those prescribed ULT, with factors included in the model with $\mathrm{p}<0.15$ in the univariate analysis. The measures of gout activity were pre-specified as flare frequency, presence of tophi, work absences, and activity limitation as assessed by the HAQ-II. All tests were two tailed and $\mathrm{P}<0.05$ was considered statistically significant.

\section{Results}

\section{SU target and clinical characteristics}

A total of 273 patients attended the study visit. There were $89(32.6 \%)$ patients with serum urate level $<0.36 \mathrm{mmol} / \mathrm{L}$ ('at SU target' group). The clinical characteristics are shown for all patients in Table 1. Patients were predominantly middle-aged men treated in primary care. ULT was prescribed in 181 (66.3\%) of the entire group. Allopurinol was the most frequently used ULT, in 177 patients. Four patients received probenecid monotherapy, and no patients were taking febuxostat, benzbromarone or recombinant uricase. In univariate analysis, SU target was associated with female sex, prescription of ULT, and allopurinol dose (Table 1). No relationship was observed between SU target and disease duration, body mass index, diuretic use, serum creatinine, or creatinine clearance. Anti-inflammatory treatments such as colchicine or non-steroidal anti-inflammatory drugs were not associated with SU targets, and rates of secondary care management were not different between groups.

\section{Psychological measures, health-related behaviours and SU target}

Although there was no difference between groups in confidence to follow dietary advice, patients at SU target reported greater confidence to take gout medications regularly and to keep SU concentrations under control (Table 2). The perceived ability of medications to control gout (BIPQ treatment control score) and understanding of disease (BIPQ understanding score) were higher in those at SU target, compared with those not at target (Table 2). Other illness perception scores and healthrelated behaviours did not differ between the two groups (Table 2 and data not shown). In univariate analysis of those prescribed ULT, adherence scores were higher in patients at SU target, compared to those who were not at SU target (Table 2).

\section{Clinical and psychological factors independently associated with SU target}

The factors independently associated with SU target were determined using logistic regression analysis. All factors with $\mathrm{p}<0.15$ in the univariate analysis were included in the models. In those patients prescribed ULT $(\mathrm{n}=181)$, allopurinol dose, patient confidence to keep SU under control, female sex, and ethnicity were independently associated with SU target (Table 3). Adherence scores, other self-efficacy measures and illness perception scores were not independently associated with SU target. 
Table 1 Clinical characteristics

\begin{tabular}{|c|c|c|c|}
\hline & \multirow{2}{*}{$\frac{\text { At SU target }(<0.36 \mathrm{mmol} / \mathrm{L})}{\mathrm{n}=89}$} & \multirow{2}{*}{$\frac{\text { Above SU target }(\geq 0.36 \mathrm{mmol} / \mathrm{L})}{\mathrm{n}=184}$} & \multirow[t]{2}{*}{$p$} \\
\hline & & & \\
\hline Age, years, mean (SD) & $61(14)$ & $58(16)$ & 0.09 \\
\hline Male sex, n (\%) & $52(58 \%)$ & $143(77.7 \%)$ & 0.002 \\
\hline Māori or Pacific ethnicity, n (\%) & $15(17 \%)$ & $49(26.6 \%)$ & 0.09 \\
\hline Disease duration, years, mean (SD) & $5.6(4.8)$ & $5.0(3.0)$ & 0.22 \\
\hline Treatment in secondary care, n (\%) & $16(18 \%)$ & $52(28.2 \%)$ & 0.07 \\
\hline Body mass index, $\mathrm{kg} / \mathrm{m}^{2}$, mean (SD) & $30.4(6.8)$ & $31.6(6.5)$ & 0.16 \\
\hline Diuretic use, n (\%) & $26(29 \%)$ & $54(29.3 \%)$ & 1.0 \\
\hline Any ULT, n (\%) & $74(83 \%)$ & $107(58.1 \%)$ & $<0.001$ \\
\hline Allopurinol use, n (\%) & $74(83 \%)$ & $103(56.0 \%)$ & $<0.001$ \\
\hline Allopurinol dose for patients on allopurinol, mg/day, mean (SD) & $235(87)$ & $194(98)$ & 0.004 \\
\hline Probenecid use, n (\%) & $2(2 \%)$ & $6(3.2 \%)$ & 1.0 \\
\hline Colchicine use, n (\%) & $26(31 \%)$ & $61(33.2 \%)$ & 0.58 \\
\hline Non-steroidal anti-inflammatory use, n (\%) & $14(16 \%)$ & $43(23.4 \%)$ & 0.16 \\
\hline Serum urate, $\mathrm{mmol} / \mathrm{L}$, mean (SD) & $0.27(0.07)$ & $0.47(0.09)$ & $<0.001$ \\
\hline Serum creatinine, $\mu \mathrm{mol} / \mathrm{l}$, mean (SD) & $99(77)$ & $110(62)$ & 0.23 \\
\hline Creatinine clearance, $\mathrm{ml} / \mathrm{min}$, mean (SD) & $68(27)$ & $70(30)$ & 0.51 \\
\hline
\end{tabular}

\section{SU target and measures of gout activity}

Patients at SU target reported lower gout flare frequency $(\mathrm{p}=0.03)$, compared with those who were not at target (Table 4). There was a trend to less tophaceous disease in the group at SU target $(\mathrm{p}=0.08)$. There were no differences between groups in HAQ-II scores or work absences.

\section{Discussion}

Despite widespread recognition that long term SU lowering to target is required for good clinical outcomes in gout, this study has confirmed that the majority of patients with gout are not at SU target. This work has highlighted ULT prescription and allopurinol dosing as central modifiable factors associated with SU target. The data emphasize the important role of the health care professional to ensure ULT prescription at sufficient doses to reduce SU to target.

The finding that prescription and dose of allopurinol are key variables associated with SU target is consistent with previous reports from our group (in a different study population of patients with longstanding gout) and from others, showing a close relationship between allopurinol doses and SU concentrations [8,9]. Collectively, these findings provide further support for a treat-to-SU-target approach to long term allopurinol dosing [19]. A recent qualitative study of health care providers has identified barriers to effective gout management which include lack of knowledge about gout and reluctance to offer ULT as a long-term management strategy [20]. Furthermore, a 'package of care' intervention with close monitoring by nurse practitioners aimed to achieve serum urate target

Table 2 Psychological measures, health-related behaviours and SU target

\begin{tabular}{|c|c|c|c|}
\hline & At SU target $(<0.36 \mathrm{mmol} / \mathrm{L})$ & Above SU target $(\geq 0.36 \mathrm{mmol} / \mathrm{L})$ & $\mathbf{p}$ \\
\hline & $\mathrm{n}=89$ & $\mathrm{n}=184$ & \\
\hline Confidence to keep serum urate under control, mean (SD) & $7.4(2.4)$ & $6.7(2.7)$ & 0.02 \\
\hline Confidence to follow diet, mean (SD) & $7.5(2.3)$ & $7.4(2.3)$ & 0.76 \\
\hline Confidence to have blood tests at recommended frequency, mean (SD) & $9.4(1.1)$ & $9.0(2.0)$ & 0.06 \\
\hline Confidence to take gout medications regularly, mean (SD) & $9.5(1.4)$ & $9.0(1.7)$ & 0.03 \\
\hline BIPQ treatment control score, mean (SD) & $8.2(2.4)$ & $7.4(2.8)$ & 0.02 \\
\hline BIPQ personal control score, mean (SD) & $6.1(3.1)$ & $5.5(3.1)$ & 0.18 \\
\hline BIPQ understanding score, mean (SD) & $7.3(2.6)$ & $6.5(2.8)$ & 0.04 \\
\hline Adherence score (those on ULT only), mean (SD) & $42.7(2.7)$ & $39.0(7.4)$ & $<0.001$ \\
\hline
\end{tabular}

BIPQ: brief illness perception questionnaire. 
Table 3 Forward stepwise logistic regression analysis of factors associated with SU target in those taking ULT

\begin{tabular}{lcccc}
\hline Variable & Odds ratio & $\mathbf{9 5 \%} \mathbf{C l}$ & $\mathbf{p}$ & Model \\
\hline Female sex & 4.3 & $1.6-11.4$ & 0.003 & Adjusted $\mathrm{R}^{2}=0.35, \mathrm{p}<0.001$ \\
Māori or Pacific ethnicity & 0.19 & $0.07-0.52$ & 0.001 & \\
Allopurinol dose (per every $100 \mathrm{mg} /$ day) & 2.22 & $1.43-3.44$ & $<0.001$ \\
Confidence to keep serum urate under control & 1.025 & $1.007-1.044$ & 0.006 \\
(per every point on 0-10 Likert scale) & & & \\
\hline
\end{tabular}

Excluded from model: secondary care treatment, adherence score, age, confidence to have blood tests at recommended frequency, confidence to take gout medications regularly, BIPQ treatment control score, BIPQ understanding score.

Model included all factors with $\mathrm{p}<0.15$ between groups in univariate analysis.

has shown excellent results in patients with gout [21]. Our data further highlight the need for education of health care professionals and changes in prescriber behaviour to optimize gout management.

Many recent studies and commentaries have focused on the need to address patient factors such as adherence and health related behaviours for optimal treatment of gout [10,22-24]. However, in our analysis of patients taking ULT, adherence was not an independent variable in the logistic regression model which included allopurinol dose. Furthermore, aside from confidence to keep SU under control, patient psychological factors and healthrelated behaviours were not independently associated with SU target in those taking ULT. These data do not discount the potential role of patient behaviours, but highlight the dominant role of effective ULT prescribing.

The cross-sectional design of this study does not allow conclusions to be made about the direction of the relationship between SU target and confidence to keep SU under control; that is, whether high confidence to keep SU under control reflects the patient's experience of good control, or influences the ability to achieve target. It is possible that feedback from clinic visits in patients achieving SU target increases confidence and reinforces behaviour. Longitudinal analysis of this group is underway to further address this issue.

This study has also identified several independent non-modifiable variables associated with SU target. Women were more likely to be at target. It is possible that allopurinol doses relative to creatinine clearance may have been higher in women. However, we did not observe a relationship between creatinine clearance and SU targets in this study. Patients of Māori or Pacific ethnicity were less likely to be at target. Population based studies have demonstrated that men and those of Polynesian ancestry have higher mean SU concentrations $[25,26]$, and high baseline levels may mean that therapeutic SU targets are more difficult to achieve. Alternatively, different health-care utilisation behaviour between different sexes or ethnicities may have contributed to the differences observed. Of interest, other clinical variables associated with higher SU concentrations such as diuretic use, body mass index and creatinine clearance were not associated with SU target.

We acknowledge the potential limitations of this study. Long-term SU lowering is recommended for optimal gout management, and this study has addressed SU target at a single timepoint. Furthermore, the crosssectional nature of this study does not allow analysis of the direction of the relationship between SU target and other variables. However, many of the variables included in the analysis are modifiable and dynamic, and this approach allowed for direct analysis of these variables at the time that the SU was obtained. Although patients were recruited from a wide range of clinical settings, adherence and health-related behaviours may be different in those willing to participate in a research study, compared with those who are not. The study design is not able to capture an individual physician's reasons for not escalating doses of ULT, emphasizing the importance of future studies examining why physicians do not initiate ULT or titrate appropriately. Consistent with other studies in gout, flare frequency was self-reported, and not verified by a health care professional. However, this definition is consistent with that used in other long term studies of gout $[2,3]$.

Table 4 Serum urate target and measures of gout activity

\begin{tabular}{|c|c|c|c|}
\hline & At SU target $(<0.36 \mathrm{mmol} / \mathrm{L})$ & Above SU target $(\geq 0.36 \mathrm{mmol} / \mathrm{L})$ & $\mathbf{p}$ \\
\hline & $\overline{n=89}$ & $n=184$ & \\
\hline Flare frequency, mean (SD) & $1.2(2.3)$ & $3.2(11.4)$ & 0.03 \\
\hline Presence of tophi, n (\%) & $9(10 \%)$ & $34(18.5 \%)$ & 0.08 \\
\hline HAQ-II, mean (SD) & $0.46(0.52)$ & $0.51(0.63)$ & 0.46 \\
\hline Days off work due to gout, mean (SD) & $1.2(5.6)$ & 0.9 (3.3) & 0.52 \\
\hline
\end{tabular}




\section{Conclusions}

In summary, SU target is frequently not achieved in patients with gout. Greater ULT use and higher doses of allopurinol, rather than patient behaviours, are the key modifiable variables associated with suppression of SU to target. These data support interventions focusing on prescription and dosing of ULT to improve outcomes in patients with gout.

\section{Competing interest}

The authors have no conflict of interest to declare.

\section{Authors' contributions}

ND (the guarantor) accepts full responsibility for the work and the conduct of the study, had access to the data, and controlled the decision to publish. ND conceived the study, participated in data analysis, and drafted the manuscript, MEH and $\mathrm{AH}$ recruited the patients, collected data, and assisted with the analysis, KJP assisted with study design, data analysis, and drafting the manuscript, FMM assisted with study design and drafting the manuscript, WJT assisted in study design, data analysis, and drafting the manuscript. All authors read and approved the final manuscript.

\section{Funding statement}

This work was funded by the Arthritis New Zealand, the University of Auckland and the Henry Cotton Charitable Trust.

\section{Author details}

${ }^{1}$ Department of Medicine, Faculty of Medical and Health Sciences, University of Auckland, 85 Park RdGrafton, Auckland 1023, New Zealand. ${ }^{2}$ Department of Psychological Medicine, University of Auckland, Auckland 1023, New Zealand. ${ }^{3}$ Department of Molecular Medicine, University of Auckland, Auckland 1023, New Zealand. ${ }^{4}$ Department of Medicine, University of Otago Wellington, PO Box 7343, Wellington 6242, New Zealand.

Received: 27 July 2012 Accepted: 12 September 2012 Published: 16 September 2012

\section{References}

1. Perez-Ruiz F: Treating to target: a strategy to cure gout. Rheumatology (Oxford) 2009, 48(2):9-14

2. Li-Yu J, Clayburne G, Sieck M, Beutler A, Rull M, Eisner E, Schumacher HR Jr: Treatment of chronic gout. Can we determine when urate stores are depleted enough to prevent attacks of gout? I Rheumatol 2001, 28:577-580.

3. Shoji A, Yamanaka H, Kamatani N: A retrospective study of the relationship between serum urate level and recurrent attacks of gouty arthritis: evidence for reduction of recurrent gouty arthritis with antihyperuricemic therapy. Arthritis Rheum 2004, 51:321-325.

4. Perez-Ruiz F, Calabozo M, Pijoan Jl, Herrero-Beites AM, Ruibal A: Effect of urate-lowering therapy on the velocity of size reduction of tophi in chronic gout. Arthritis Rheum 2002, 47:356-360.

5. Perez-Ruiz F, Martin I, Canteli B: Ultrasonographic measurement of tophi as an outcome measure for chronic gout. J Rheumatol 2007, 34:1888-1893.

6. Jordan KM, Cameron JS, Snaith M, Zhang W, Doherty M, Seckl J, Hingorani A, Jaques R, Nuki G: British Society for Rheumatology and British Health Professionals in Rheumatology guideline for the management of gout. Rheumatology (Oxford) 2007, 46:1372-1374.

7. Zhang W, Doherty M, Bardin T, Pascual E, Barskova V, Conaghan P, Gerster J, Jacobs J, Leeb B, Liote F, McCarthy G, Netter P, Nuki G, Perez-Ruiz F, Pignone A, Pimentao J, Punzi L, Roddy E, Uhlig T, Zimmermann-Gorska I: EULAR evidence based recommendations for gout. Part II: Management. Report of a task force of the EULAR Standing Committee for International Clinical Studies Including Therapeutics (ESCISIT). Ann Rheum Dis 2006, 65:1312-1324.

8. Dalbeth N, Kumar S, Stamp L, Gow P: Dose adjustment of allopurinol according to creatinine clearance does not provide adequate control of hyperuricemia in patients with gout. J Rheumatol 2006, 33:1646-1650.
9. Roddy E, Zhang W, Doherty M: Concordance of the management of chronic gout in a UK primary-care population with the EULAR gout recommendations. Ann Rheum Dis 2007, 66:1311-1315.

10. Harrold LR, Andrade SE, Briesacher BA, Raebel MA, Fouayzi H, Yood RA, Ockene IS: Adherence with urate-lowering therapies for the treatment of gout. Arthritis Res Ther 2009, 11:R46.

11. Mikuls TR, Farrar JT, Bilker WB, Fernandes S, Saag KG: Suboptimal physician adherence to quality indicators for the management of gout and asymptomatic hyperuricaemia: results from the UK General Practice Research Database (GPRD). Rheumatology (Oxford) 2005, 44:1038-1042.

12. Edwards NL, Quality of Care in Patients with Gout: Why is Management Suboptimal and What Can Be Done About It? Curr Rheumatol Rep: Curr Rheumatol Rep; 2010.

13. Harrold LR, Mazor KM, Velten S, Ockene IS, Yood RA: Patients and providers view gout differently: a qualitative study. Chronic IIIn 2010, 6:263-271.

14. Solomon DH, Avorn J, Levin R, Brookhart MA: Uric acid lowering therapy: prescribing patterns in a large cohort of older adults. Ann Rheum Dis 2008, 67:609-613.

15. Suppiah R, Dissanayake A, Dalbeth $\mathrm{N}$ : High prevalence of gout in patients with Type 2 diabetes: male sex, renal impairment, and diuretic use are major risk factors. N Z Med J 2008, 121:43-50.

16. Wallace SL, Robinson H, Masi AT, Decker JL, McCarty DJ, Yu TF: Preliminary criteria for the classification of the acute arthritis of primary gout. Arthritis Rheum 1977, 20:895-900.

17. Dalbeth N, Petrie K, House M, Chong J, Leung W, Chegudi R, Horne A, Gamble G, McQueen FM, Taylor WJ: Illness perceptions in patients with gout and the relationship with progression of musculoskeletal disability. Arthritis Care Res (Hoboken) 2011, 63:1605-1612.

18. Talbot F, Nouwen A, Gingras J, Gosselin M, Audet J: The assessment of diabetes-related cognitive and social factors: the Multidimensional Diabetes Questionnaire. J Behav Med 1997, 20:291-312.

19. Stamp LK, O'Donnell JL, Zhang M, James J, Frampton C, Barclay ML, Chapman PT: Using allopurinol above the dose based on creatinine clearance is effective and safe in patients with chronic gout, including those with renal impairment. Arthritis Rheum 2011, 63:412-421.

20. Spencer K, Carr A, Doherty M: Patient and provider barriers to effective management of gout in general practice: a qualitative study. Ann Rheum Dis 2012.

21. Rees F, Jenkins W, Doherty M: Patients with gout adhere to curative treatment if informed appropriately: proof-of-concept observational study. Ann Rheum Dis 2012

22. Chandratre $P$, Roddy E, Mallen C: Patient related factors are also important in treating gout. BMJ 2012, 344:e191.

23. Dalbeth $\mathrm{N}$, Lindsay $\mathrm{K}$ : The patient's experience of gout: new insights to optimize management. Curr Rheumatol Rep 2012, 14:173-178.

24. Reach G: Treatment adherence in patients with gout. Joint Bone Spine 2011, 78:456-459.

25. Zhu Y, Pandya BJ, Choi HK: Prevalence of gout and hyperuricemia in the US general population: the National Health and Nutrition Examination Survey 2007-2008. Arthritis Rheum 2011, 63:3136-3141.

26. Prior IA, Rose BS, Harvey HP, Davidson F: Hyperuricaemia, gout, and diabetic abnormality in Polynesian people. Lancet 1966, 1:333-338.

doi:10.1186/1471-2474-13-174

Cite this article as: Dalbeth et al:: Prescription and dosing of uratelowering therapy, rather than patient behaviours, are the key modifiable factors associated with targeting serum urate in gout. BMC Musculoskeletal Disorders 2012 13:174. 